\title{
RAPID IMPLEMENTATION OF TELEREHABILITATION FOR PEDIATRIC PATIENTS DURING COVID-19
}

\author{
RACHEL BICAN, PT, DPT ${ }^{1,2}$, CATIE CHRISTENSEN, PT, DPT, PCS ${ }^{1}$, KRISTIN \\ FALLIERAS, PT ${ }^{1}$, GRACE SAGESTER, OTD, OTR/L ${ }^{1}$, SARA O’ROURKE, MOT, OTR/L, \\ $\mathrm{BCP}^{1}$, MICHELLE BYARS, $\mathrm{PT}^{1}$, KELLY TANNER, PHD, OTR/L, BCP \\ ${ }^{1}$ DIVISION OF CLINICAL THERAPIES, NATIONWIDE CHILDREN'S HOSPITAL, COLUMBUS, OHIO, USA \\ ${ }^{2}$ SCHOOL OF HEALTH AND REHABILITATION SCIENCES, COLLEGE OF MEDICINE, THE OHIO STATE UNIVERSITY, \\ COLUMBUS, OHIO, USA
}

\begin{abstract}
The COVID-19 pandemic necessitated a sudden limitation of in-person outpatient occupational and physical therapy services for most patients at a large, multisite pediatric hospital located in the Midwest, United States. To ensure patient and staff safety, the hospital rapidly shifted to deliver most of these services via telerehabilitation. The purposes of this study were to (1) describe the rapid implementation of telerehabilitation during the COVID-19 pandemic, (2) describe the demographic characteristics of patients who continued in-person services and those who received telerehabilitation, and (3) evaluate the therapists' perceptions of telerehabilitation for physical and occupational therapy. Most of the children (83.4\% of $n=1352$ ) received telerehabilitation services. A family was more likely to choose to continue in-person visits if their child was <1-year-old, had a diagnosis of torticollis, received serial casting, or was post-surgical. Occupational and physical therapy therapists $(n=9)$ completed surveys to discern their perceptions of the acceptability of telerehabilitation, with most reporting that telerehabilitation was as effective as in-person care.
\end{abstract}

Keywords: Covid-19, Occupational Therapy, Pediatrics, Physical Therapy, Telehealth, Telerehabilitation

Telerehabilitation has been proposed as a potentially effective model of care for pediatric populations (Camden et al., 2020; Olson et al., 2018; Shigekawa et al., 2018; Tanner et al., 2020; Tenforde et al., 2017, 2020). However, barriers to implementation, including payor reimbursement, perceived and actual technology barriers, liability concerns, and privacy concerns, have prevented routine adoption of this service delivery model (Brophy, 2017; Dorsey \& Topol, 2016; Lee et al., 2018; Olson et al., 2018; Sauers-Ford et al., 2019; Tomines, 2019). The Covid-19 pandemic, a public health crisis, accelerated the use of telerehabilitation for pediatric populations to adhere to social distancing guidelines, prevent disruption to an established plan of care, and ensure access to essential therapeutic services (Badawy \& Radovic, 2020; Ben-Pazi et al., 2020; Burke et al., 2015; Tomines, 2019).

Previous literature has shown telerehabilitation can be utilized to provide outpatient services for pediatric and adult patients and can be an effective, efficient, and affordable model of care (Burke et al., 2015; Cramer et al., 2019; Levy et al., 2015; Olson et al., 2018; Tenforde et al., 2020). The American Occupational Therapy Association and American Physical Therapy Association both support the use of telerehabilitation (i.e., telehealth) for clinical care, stating that telerehabilitation can be effective in improving patient outcomes and can be flexibly delivered to meet the needs of the patients and their families (American Occupational Therapy Association, 2018; American Physical Therapy Association, 2019).

The effectiveness and applicability of telerehabilitation may vary by pediatric specialty, setting, and patient preference (Tomines, 2019). Studies have shown that telerehabilitation may be effective for pediatric rehabilitation, primary care, and mental health treatment (Gloff et al., 2015; Nelson \& Sharp, 2016; Tomines, 2019). Telerehabilitation has also been shown to be feasible and effective in treating children with cerebral palsy (Reifenberg et al., 2017) and autism spectrum disorder (Little et al., 2018). However, since telerehabilitation has not been routinely adopted for pediatric occupational and physical therapies, there is currently no consensus on which patient populations may best be served by telerehabilitation throughout or beyond the COVID-19 pandemic. 
The COVID-19 pandemic led to a sudden limitation of in-person outpatient therapy services for most patients at Nationwide Children's Hospital (Tanner et al., 2020). To ensure patient and staff safety, the hospital had to rapidly and unexpectedly prepare to deliver these services via telerehabilitation (Tanner et al., 2020). Therefore, the purposes of this study were to (1) describe the rapid implementation of telerehabilitation during the COVID-19 pandemic, (2) describe the demographic characteristics of patients who continued in-person services and those who received telerehabilitation, and (3) evaluate the therapists' perceptions of telerehabilitation for physical and occupational therapy at a large, multisite pediatric hospital located in the Midwest, United States.

\section{METHODS}

\section{PARTICIPANTS}

The procedures for this retrospective, observational study were approved by the Institutional Review Board of Nationwide Children's Hospital (STUDY00001188). All patients receiving developmental occupational or physical therapy at Nationwide Children's Hospital, a large, urban, multisite pediatric hospital system, between April 1, 2020 and April 30, 2020 were included in this study. Of note, a stay-at-home order was issued for the state of Ohio on March 18, 2020 in response to the COVID-19 pandemic. During this time, both in-person clinic visits and telerehabilitation were available. Patients were recommended for in-person clinic visits if they met one or more of the following criteria: (1) less than one year old, (2) had torticollis, (3) were currently receiving, or were planning to receive, serial casting, (4) had recently been discharged from inpatient rehabilitation or had surgery, or (5) had no access to technology required for telerehabilitation. These criteria were developed by the

Developmental Physical and Occupational Therapy Program Managers with input via email from all staff members. For patients that met the criteria for in-person clinic visits, families ultimately were able to decide whether to continue in-person visits or transition to a telerehabilitation service delivery model. If a child did not meet the criteria for in-person visits, they were offered services through telerehabilitation or placed on-hold based on family preference.

\section{PROCEDURES}

\section{IMPLEMENTATION OF TELEHEALTH}

Over a three-week period, the hospital developed and implemented a strategic plan for the rapid uptake of services provided through telerehabilitation, including preparation for telerehabilitation and telerehabilitation roll-out to ensure a successful transition from an in-person to a telerehabilitation service delivery model. This rapid implementation was carried out by a multidisciplinary team that included the director of rehabilitation services, program managers, clinical leaders, performance improvement staff, evidence-based practice and research coordinators, information technology staff, scheduling support staff, physical and occupational therapists, and therapy aides.

\section{PREPARATION FOR TELEREHABILITATION}

The first component for telerehabilitation preparation was to obtain, organize, and train all staff on technology and documentation. The hospital developed two methods of telerehabilitation service delivery: billable telephone calls and video sessions via a secure Zoom video platform (Zoom, 2020). Telephone calls were completed through the therapist's work phone. Video telerehabilitation occurred through Zoom, a web-based video conferencing program, hosted by Epic Systems (Software | Epic, n.d.), the electronic medical records system utilized by the hospital. The sessions were Health Insurance Portability and Accountability Act (HIPAA) compliant, encrypted, and password protected. All telerehabilitation documentation included caregiver consent and followed payor guidelines.

Next, the hospital developed specific messaging to caregivers and families regarding telerehabilitation. The treating therapist initiated the first contact with the family via phone with the goals to provide information on the upcoming switch to telerehabilitation, determine if the family was willing to participate, and decide the most appropriate method of telerehabilitation service delivery according to the family's needs. A written script was developed to guide therapists in this discussion with 
families. Parents were provided with background information on telerehabilitation, supporting evidence for this service delivery model, and details on how it may be customized for each child.

Finally, all staff members were educated on telerehabilitation best practices based on the available literature, using both formal and informal methods. Formal methods included mandatory in-service training, journal clubs, and shared resources. Informally, staff members were encouraged to ask questions and provide feedback via email and in-person. This staff education emphasized normalizing services provided through telerehabilitation, highlighting research on telerehabilitation, providing a step-by-step guide for conducting a telerehabilitation therapy session, identifying the location of additional resources, and having adequate time for discussion and case examples. Staff education was provided in the two weeks prior to initiating services through telerehabilitation, as well as on an ongoing basis during the delivery of these services.

\section{TELEREHABILITATION ROLL-OUT}

The leadership team at the hospital prioritized the rapid uptake of services through telerehabilitation to promote the safety and well-being of patients, families, and staff during a public health crisis, as well as to minimize any lapses in meaningful and therapeutically necessary patient care.

These goals allowed the department to support therapists to refocus their treatment sessions using the World Health Organization's International Classification of Functioning, Disability, and Health to prioritize the domains of Activities and Participation, rather than Body Functions and Structures (WHO I International Classification of Functioning, Disability and Health (ICF), n.d.). This framework emphasized the prioritization of functional, participation-based goals to allow therapists to provide meaningful services in the child's natural environment.

When we first began using telerehabilitation, we shifted from providing direct patient care to using a hybrid consultation/coaching model via telerehabilitation. We emphasized a parent coaching model to support caregivers navigating new challenges to their family's disrupted daily routine, allowing flexibility for families that may not have access to technology for video visits or with a child who had difficulty attending to a therapist on a screen. Parent coaching also provided an evidence-based framework to support therapists with their transition to telerehabilitation sessions (Little et al., 2018; Baldwin et al., 2013; Novak et al., 2020). The framework for parent coaching included capitalizing on the family's authentic context by integrating the family's interests and routines in treatment sessions, fostering a relationship between the caregiver and child, building in strategies for reflection and feedback, and collaborating with the caregiver to establish joint plans (Little et al., 2018; Wallisch et al., 2019).

\section{MEASURES}

\section{PATIENT DEMOGRAPHIC INFORMATION}

Demographic information was extracted from Epic using the Information Data Enterprise Application (IDEA). IDEA gives users the ability to build queries on the appointment, visit, account, and demographic data from within the electronic medical record for analysis and reporting. Report dates were April 1, 2020 - April 30, 2020. Data extracted included the following: (1) date of visit, (2) patient age at visit (years), (3) rehabilitation type (physical or occupational therapy), (4) service delivery type (clinic or telerehabilitation), (5) diagnosis (ICD-10), (6) patient language, and (7) patient zip code and city of residence. Patient ICD-10 referral diagnoses were then categorized into 13 groups for analysis (Table 1). If a child had multiple diagnoses, the primary diagnosis was used for classification. Zip codes were classified using the 2013 National Center for Health Statistics Urban-Rural Classification Scheme for Counties (DD Ingram \& SJ Franco, n.d.). This classification includes six levels based on location to a city and population size: large central metro, large fringe metro, medium metro, small metro, micropolitan, and noncore. 
Table 1

Diagnosis Group

\begin{tabular}{|c|c|c|}
\hline $\begin{array}{l}\text { Patients } \\
(\mathrm{N})\end{array}$ & Diagnosis Group & Description of Diagnosis Group \\
\hline 207 & $\begin{array}{l}\text { Abnormalities in physiological } \\
\text { development }\end{array}$ & $\begin{array}{l}\text { Any diagnosis that involved abnormalities or delays in physiological } \\
\text { development }\end{array}$ \\
\hline 185 & Autism Spectrum Disorder & Diagnosis of autism spectrum disorder \\
\hline 74 & $\begin{array}{l}\text { Behavioral, sensory, and } \\
\text { intellectual impairment }\end{array}$ & $\begin{array}{l}\text { Any diagnosis that related to patient behavior, sensory processing, or } \\
\text { intellectual impairments }\end{array}$ \\
\hline 7 & Cardiac impairment & Any diagnosis that indicated a cardiac impairment or cardiac malformation \\
\hline 101 & Cerebral palsy & Any diagnosis that included cerebral palsy \\
\hline 144 & Congenital abnormalities & $\begin{array}{l}\text { Any diagnosis indicating congenital abnormalities including syndromes, } \\
\text { chromosomal abnormalities, and malformations }\end{array}$ \\
\hline 9 & Delayed milestones & $\begin{array}{l}\text { Any diagnosis indicating delayed developmental milestones including } \\
\text { gross motor, fine motor, and language }\end{array}$ \\
\hline 81 & Feeding difficulties & Any diagnosis that indicated feeding difficulties \\
\hline 195 & Movement disorder & $\begin{array}{l}\text { Any diagnosis of abnormal movement including hypotonia, ataxia, and } \\
\text { apraxia }\end{array}$ \\
\hline 220 & Musculoskeletal impairment & Any diagnosis that indicated impairment of the musculoskeletal system \\
\hline 50 & Non-congenital insult to the brain & $\begin{array}{l}\text { Any diagnosis that indicated a non-congenital insult to the brain, such as } \\
\text { stroke or cancer }\end{array}$ \\
\hline 44 & Other & Any diagnosis that did not fit into any of the defined diagnosis groups \\
\hline 34 & Prematurity or low birth weight & Any diagnosis that indicated a premature birth or low birth weight \\
\hline
\end{tabular}

\section{THERAPIST SURVEYS}

During the specified time frame of the study, therapists $(n=9)$ elected to work from home one day per week, during which they treated exclusively telerehabilitation patients, either via video or phone. These therapists completed a work-from-home survey at the conclusion of each remote workday. Therapists who utilized a hybrid approach while remaining on-site throughout the week were excluded from this survey process.

The survey to gauge the successes of our sessions delivered through telerehabilitation was developed by Developmental Physical and Occupational Therapy Program Managers. It was delivered to physical and occupational therapists via the Research Electronic Data Capture (REDCap) tools hosted at Nationwide Children's Hospital (Harris et al., 2009, 2019). REDCap is a secure, web-based software platform designed to support data capture for research studies, providing (1) an intuitive interface for validated data capture; (2) audit trails for tracking data manipulation and export procedures; (3) automated export procedures for seamless data downloads to common statistical packages; and (4) procedures for data integration and interoperability with external sources (Harris et al., 2019). The survey had a total of 20 questions: nine yes/no, five multiple choice, and six open-ended. The questions addressed three domains of telerehabilitation: (1) technology, (2) clinical care quality, and (3) caregiver involvement. 


\section{DATA ANALYSIS}

IBM SPSS version 23.0 was used for statistical analysis. Means, standard deviations, medians, and IQR are used to describe patient age. Percentages are used to describe diagnosis group, language preference, Centers for Disease Control (CDC) county level for all patients, and therapist survey results.

\section{RESULTS}

\section{RAPID IMPLEMENTATION OF TELEREHABILITATION}

Physical and occupational therapists were provided education on telerehabilitation at seven different outpatient rehabilitation settings within the same pediatric hospital system. From April 1, 2020 to April 30, 2020, there were a total of 1,352 unique patient visits ( $n=514$ patient visits for physical therapy; $n=838$ patient visits for occupational therapy) (Figure 1 ). Patients seen in the clinic were $n=224(16.6 \%)$ and patients seen via telerehabilitation were $n=1,128(83.4 \%)$. There were 938 video visits and 150 telephone encounters.

\section{Figure 1a}

Service Delivery Type

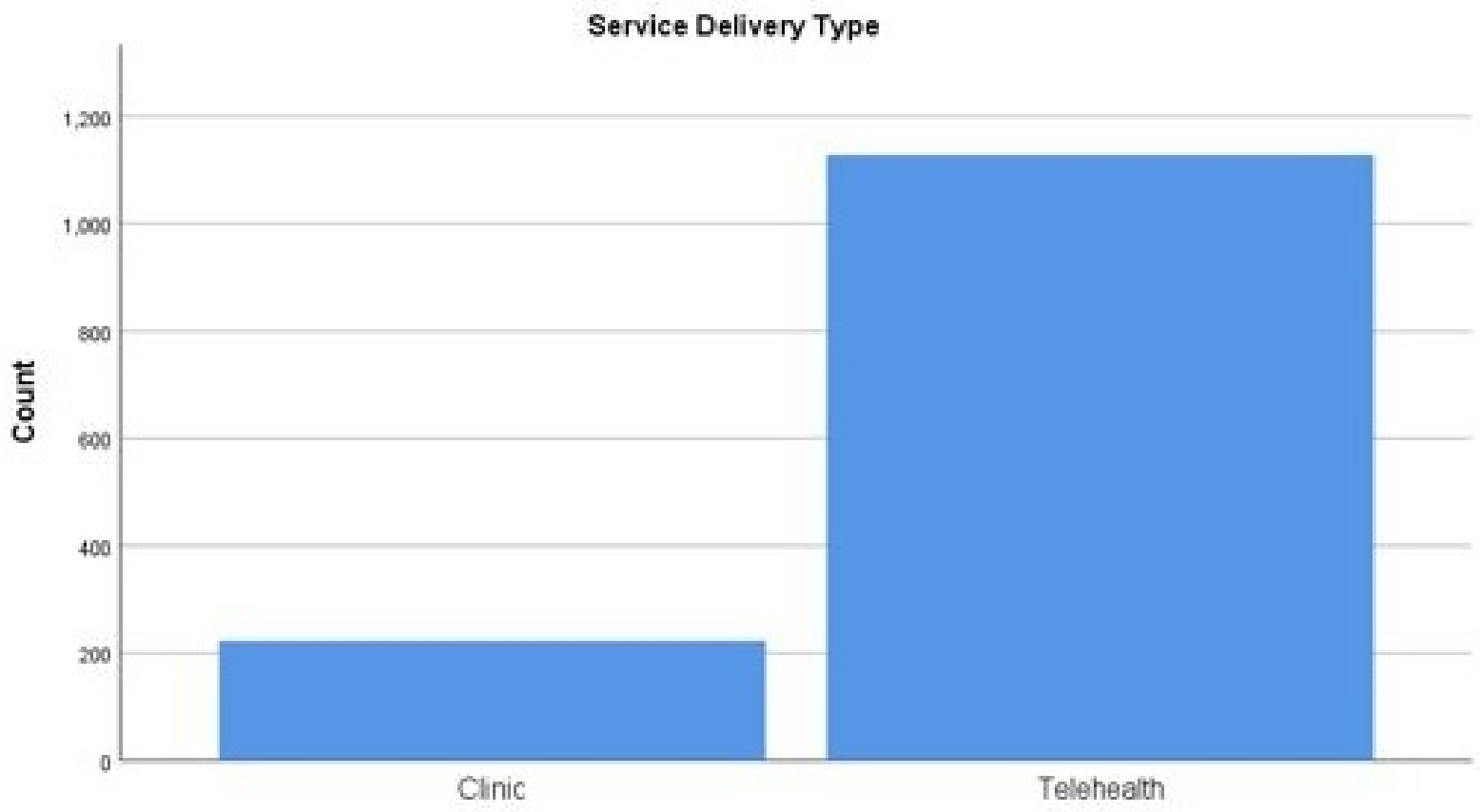

Service Delivery Type

Figure 1a. Bar graph of count of patients seen in the clinic (left) or via telerehabilitation (right). $\mathrm{n}=224$ patients were seen in the clinic and $n=1,128$ patients were seen via telerehabilitation. 
Figure $1 \mathrm{~b}$

Service Delivery Types and Patients Ages

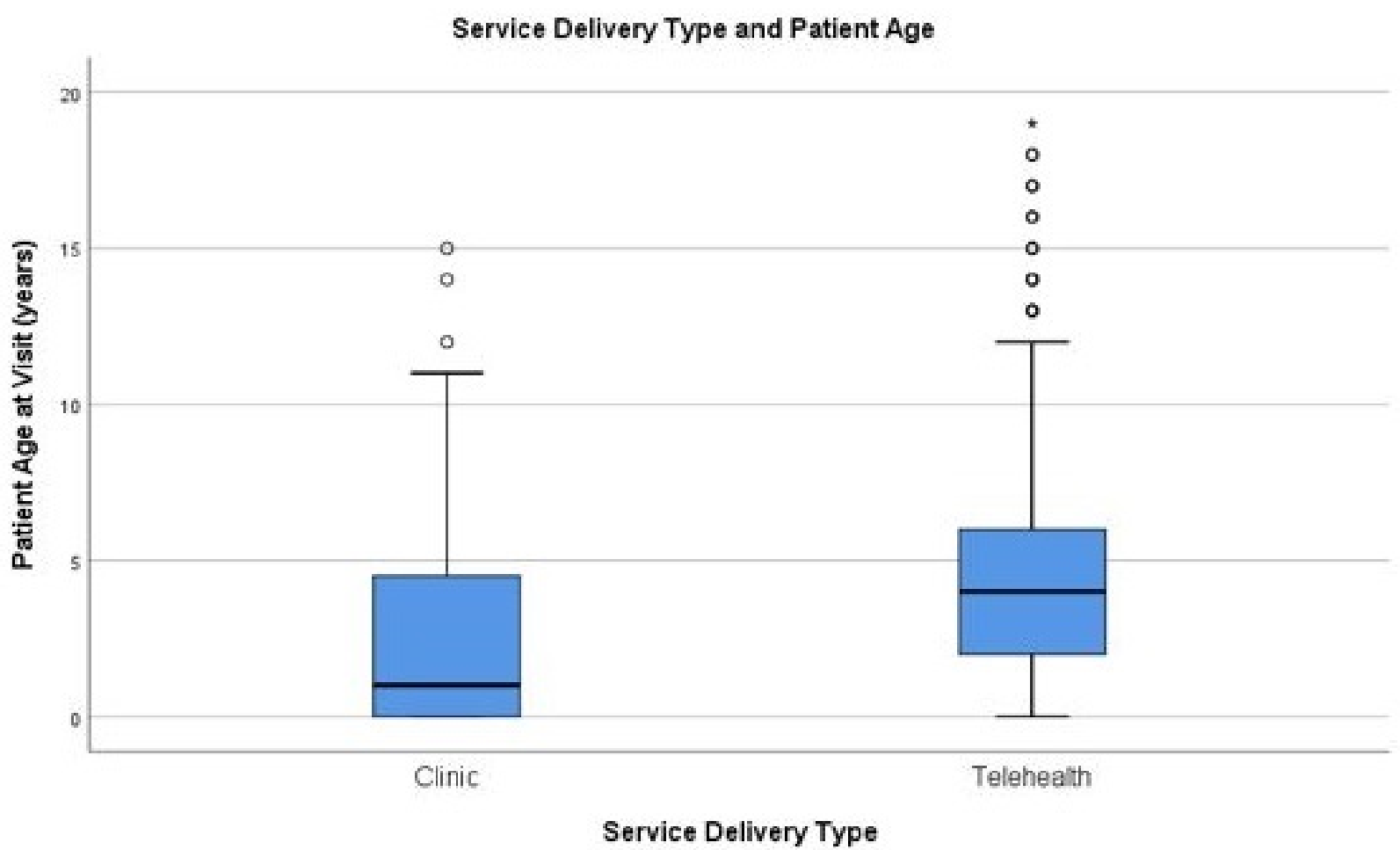

Figure $1 \mathrm{~b}$. Boxplot of service delivery type (clinic on the left or telerehabilitation on the right) by age of the patient at the time of the encounter. The median age of patients seen in the clinic was 1.0-year-old with an IQR of 5.0 years (2.5 \pm .22 years), and

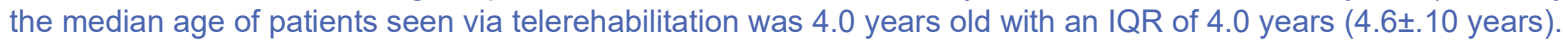

\section{DEMOGRAPHICS OF PATIENTS RECEIVING REHABILITATION SERVICES DURING COVID-19}

The median age of patients receiving physical or occupational therapy was 4.0 years old with an interquartile range (IQR) of 5.0 years $(4.3 \pm 3.5$ years) at the time of visit. The median age of patients seen in the clinic was 1.0 -year-old with an IQR of 5.0 years $(2.5 \pm .22$ years) and the median age of patients seen via telerehabilitation was 4.0 years old with an IQR of 4.0 years

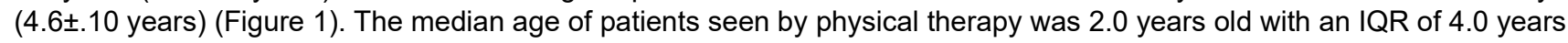

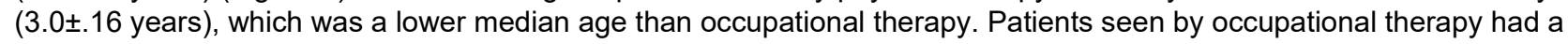

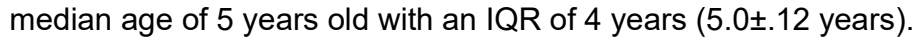

Patients with musculoskeletal impairments had the highest percentage of in-person clinic visits (41.0\%), followed by cardiac impairment (28.6\%), autism spectrum disorder $(22.2 \%)$, and delayed milestones (22.2\%). Patients with prematurity or low birth weight had the highest percentage of telerehabilitation visits (95.2\%), followed by feeding difficulties (93.1\%), behavioral, sensory, and intellectual impairment (91.8\%), and congenital abnormalities (84.0\%) (Figure 2).

The patient diagnosis group with the youngest median age was musculoskeletal impairments ( 0 years old with an IQR of 3 years; $2.06 \pm .23$ years) followed by prematurity or low birth weight (1 year-old with an IQR of 3 years; $1.65 \pm .28$ years), cardiac impairment (1-year-old with an IQR of 4 years; $2.29 \pm .55$ years). The oldest patient diagnosis group was patients with a behavioral, sensory, and intellectual impairment (7 years old with an IQR of 3 years; $7.05 \pm .18$ years old), followed by autism (5 years old with an IQR of 2 years; $5.34 \pm .18$ years old), and cerebral palsy (6 years old with an IQR of 5 years; $6.87 \pm .40$ years old) (Figure 2). 
Figure 2

Diagnosis Group by Service Delivery Type and Age
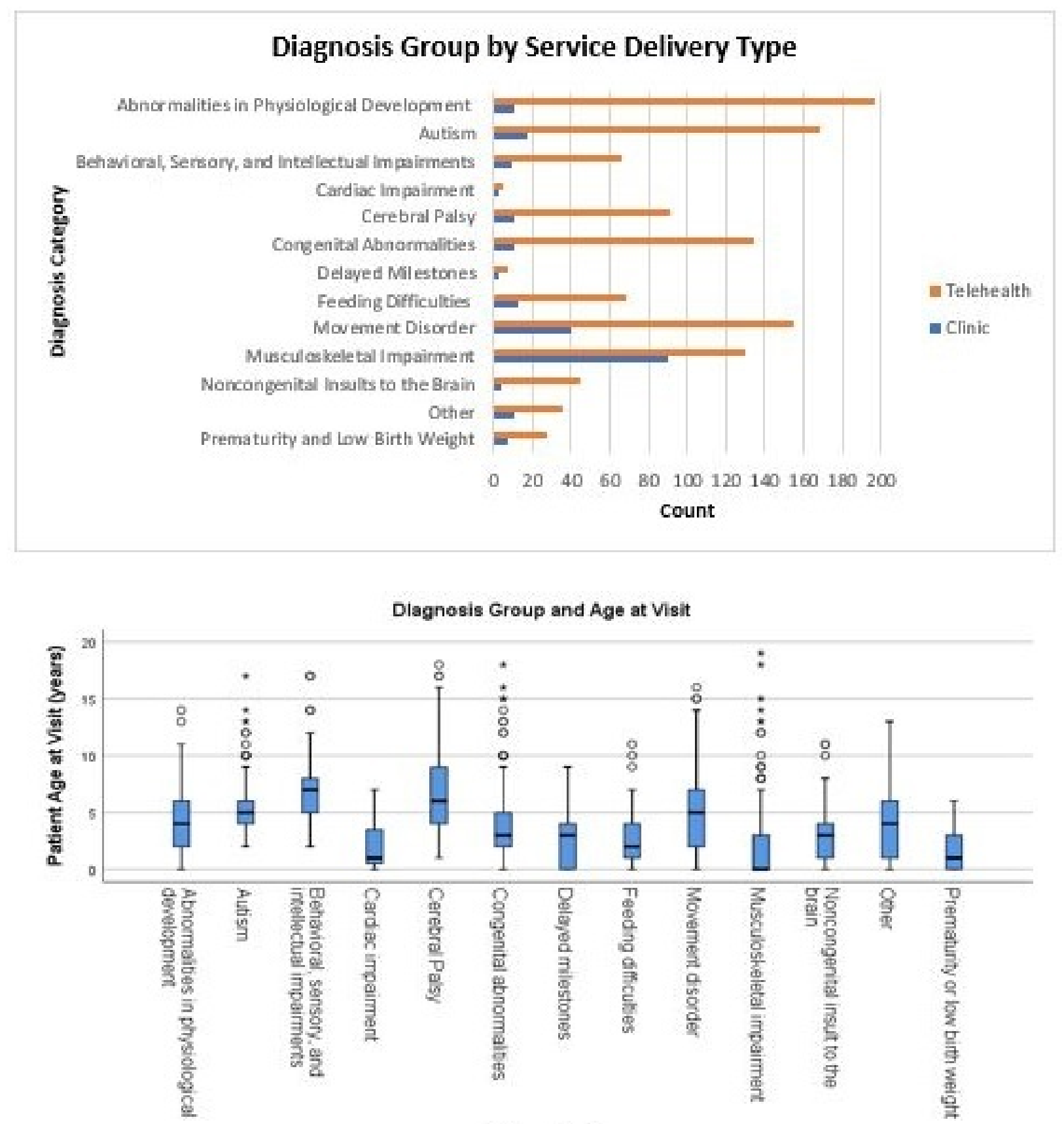

Diagnosis Group

Figure 2a. Bar graph of count of service delivery type by diagnosis group. The proportion of children in each diagnosis group receiving telerehabilitation (orange) and in-person clinical care (blue). Patients with musculoskeletal impairments had the highest percentage of in-person clinic visits (41.0\%). Patients with prematurity or low birth weight had the highest percentage of telerehabilitation visits (95.2\%). Figure $2 b$. Boxplot of diagnosis group by age. The patient diagnosis group with the youngest median age was musculoskeletal impairments (0 years old with an IQR of 3 years; $2.06 \pm .23$ years). The oldest patient diagnosis group was patients with a behavioral, sensory, and intellectual impairment (7 years old with an IQR of 3 years; $7.05 \pm .18$ years old). 
There were 20 different language preferences reported. Most patients and families in this cohort designated English as their language preference (90.9\%), followed by Spanish (3.3\%), Nepali (1.3\%), Somali (1.3\%), Arabic (0.7\%), Amharic (0.4\%), American Sign Language (0.2\%), and other (1.9\%). Of the English-speaking patients, $15.8 \%$ were seen in the clinic. Patients who spoke Somali had the highest percentage of clinic visits (38.9\%), followed by patients who spoke Nepali (29.4\%), Arabic (10.0\%), Amharic (0\%), and American Sign Language (0\%) (Figure 3a and Figure 3b).

Figure 3a

Language Preferences of Patients

\section{English and Non-English Language Preference}

1400

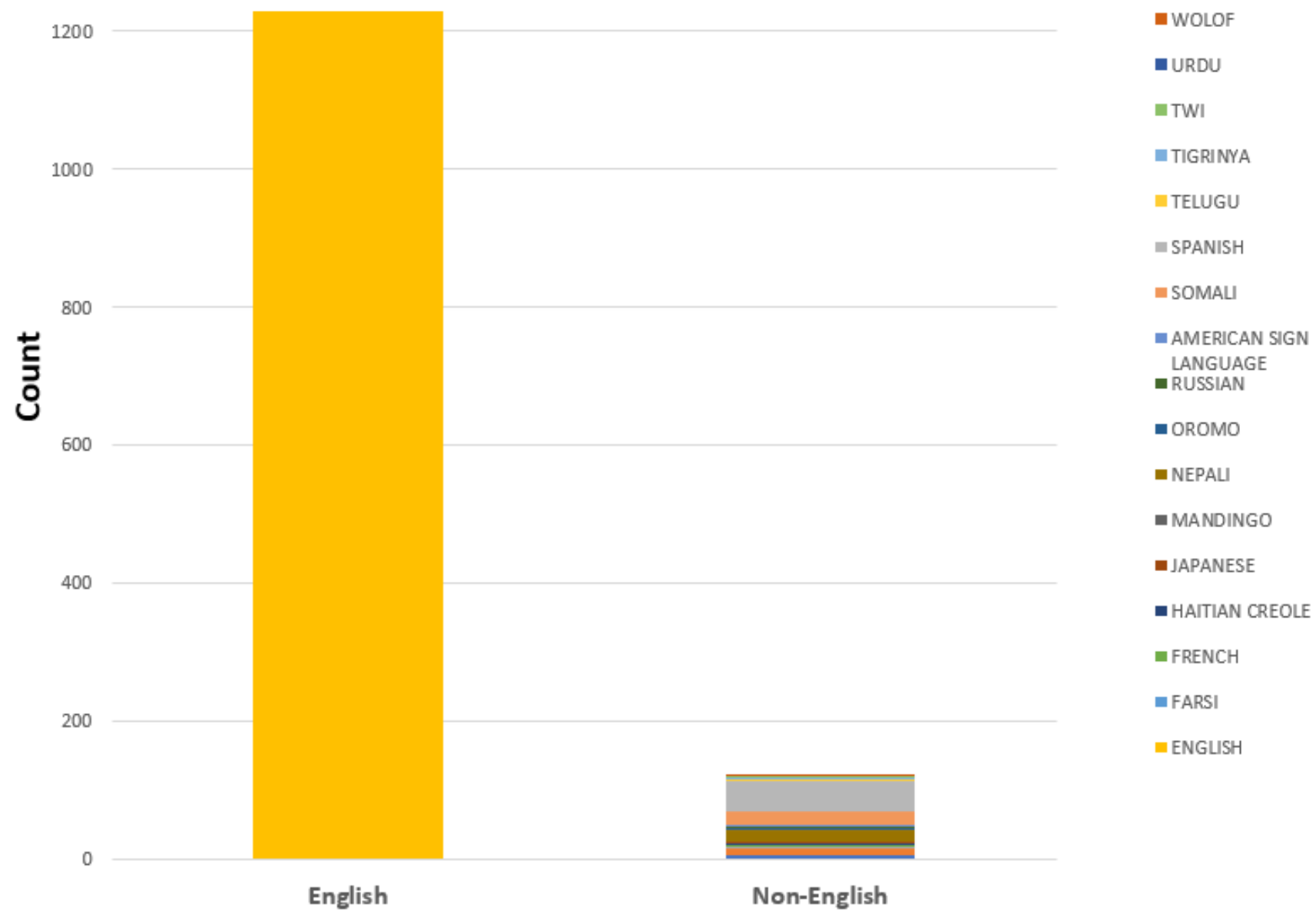

Language

Figure 3a. English (90.9\%) and non-English language (9.1\%) preferences for patients receiving services in the clinic and via telerehabilitation. 
Figure $3 b$

Patient Language (Non-English) and Service Delivery Type

\section{Patient Language (Non-English) and Service Delivery Type}

40

35

30

25

产

15

2010

20

10

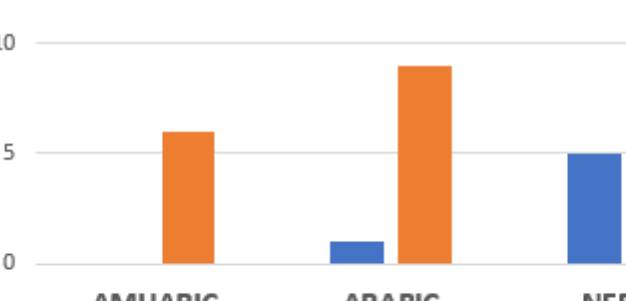

AMHARIC

ARABIC

NEPALI

AMERICAN SIGN

LANGUAGE

- Clinic

Telehealth

\section{Language}

Figure $3 b$. Most non-English speaking patients were seen via telerehabilitation.

Each of the six levels of urban-to-rural classifications was represented in this cohort. Most patients were classified as living in a large central metro $(61.0 \%)$, followed by large fringe metro (24.9\%), micropolitan $(6.9 \%)$, small metro $(6.4 \%)$, noncore $(0.5 \%)$, and medium metro $(0.2 \%)$.

\section{THERAPIST SURVEY}

The technology questions answered by the therapists are presented in Table 2. The results showed an $89.2-99.7 \%$ positive responses regarding technology. Most therapists $(69.1 \%)$ reported that telerehabilitation sessions were equal in clinical care to in-person clinic visits; $13.4 \%$ reported that telerehabilitation was better, and $17.5 \%$ reported that telerehabilitation was not equal to in-person clinic visits. In addition, most therapists (92.5\%) reported that caregivers were present and actively participating in the telerehabilitation session, $3.6 \%$ reported that caregivers were observing but not participating, and $3.9 \%$ reported caregivers were present but occupied in the background. 
Table 2

Technology Questions and Therapist Response

\begin{tabular}{|l|c|c|}
\hline \multicolumn{1}{|c|}{ Question } & Therapist response "yes" (\%) & Therapist response "no" (\%) \\
\hline $\begin{array}{l}\text { Were there any hardware challenges } \\
\text { for the clinician? }\end{array}$ & $1.9 \%$ & $98.1 \%$ \\
\hline $\begin{array}{l}\text { Were there any connectivity (WIFI) } \\
\text { challenges for the clinician? }\end{array}$ & $1.7 \%$ & $98.3 \%$ \\
\hline $\begin{array}{l}\text { Were there any application/ EPIC/ } \\
\text { MyChart challenges for the clinician? }\end{array}$ & $1.9 \%$ & $98.1 \%$ \\
\hline $\begin{array}{l}\text { Were there any hardware challenges } \\
\text { for the family? }\end{array}$ & $3.6 \%$ & $96.4 \%$ \\
\hline $\begin{array}{l}\text { Were there any connectivity (WIFI) } \\
\text { challenges for the family? }\end{array}$ & $10.8 \%$ & $89.2 \%$ \\
\hline $\begin{array}{l}\text { Were there any application/ EPIC/ } \\
\text { MyChart challenges for the family? }\end{array}$ & $6.1 \%$ & $93.9 \%$ \\
\hline $\begin{array}{l}\text { Did clinician have access to all } \\
\text { materials needed for this session? }\end{array}$ & $95.3 \%$ & $4.7 \%$ \\
\hline $\begin{array}{l}\text { Was an interpreter used during this } \\
\text { session? }\end{array}$ & $3.6 \%$ & $96.4 \%$ \\
\hline $\begin{array}{l}\text { Was an interpreter needed but not } \\
\text { obtained? }\end{array}$ & $0.3 \%$ & $99.7 \%$ \\
\hline
\end{tabular}

\section{DISCUSSION}

Rapid implementation of telerehabilitation was critical during the COVID-19 pandemic for pediatric developmental therapies to continue to provide safe and high-quality clinical services to vulnerable populations (Ben-Pazi et al., 2020). Our study sought to describe the rapid implementation of telerehabilitation, describe demographic characteristics of patients who continued in-person services and those who received telerehabilitation, and evaluate therapists' perceptions of telerehabilitation for physical and occupational therapy at a large, pediatric hospital system in the Midwest, United States.

Telerehabilitation was successfully and rapidly implemented at Nationwide Children's Hospital for developmental occupational and physical therapies. Implementation of telerehabilitation was completed through a multidisciplinary team approach that addressed (1) technology, billing, and documentation, (2) messaging to staff, caregivers, and families, (3) formal and informal educational opportunities, and (5) refinement of services provided through telerehabilitation through feedback from staff and families. Utilizing both telerehabilitation and in-person models of care, services were maintained for a diverse group of patients during the COVID-19 pandemic. Patients seen during this period were (1) 0-18 years old, (2) had 240 unique referral diagnoses, (3) had 20 different language preferences, and (4) represented all county-level populations as categorized by the CDC. These results highlight the usefulness of offering telerehabilitation to maximize patient encounters and continue vital services during a public health crisis, as well as presenting opportunities for future utilization.

Most patients were seen through telerehabilitation (83.4\%) at our institution in the early months of the COVID-19 pandemic. Some patients did continue with in-person clinical care; specifically, younger patients and patients with musculoskeletal conditions were more often seen in the clinic. This likely occurred due to our hospital therapy recommendations of who should continue in-person clinical care. These recommendations were developed based on clinical expertise, suggesting that patients under 1-year-old, with torticollis, undergoing serial casting, without access to technology, or who were post-surgery should continue in-person clinical care. We propose, based on clinical expertise that these patients may benefit from continued in-person clinical care because hands-on therapeutic techniques are deemed critical for successful treatment. 
Although our recommendations for in-person clinical care were for all patients less than 1-year-old, patients with prematurity or low birth weight (median age of 1-year) had the highest percentage of telerehabilitation visits (95.2\%). This population is especially vulnerable in the first year of life, and caregivers/families may have opted for telerehabilitation to promote safety and social distancing. This finding emphasizes the importance of allowing the decision for the model of care to be determined by the caregivers and families of the patients. Parents and families should be educated regarding benefits of and barriers to telerehabilitation to assist in making the decision for the best model of care for their child.

Of the patients seen in-person, more spoke Somali or Nepali than English. No other confounding factors were controlled for, so we cannot say whether these patients were seen in the clinic due to their language preference. However, it is important to consider language when implementing services through telerehabilitation and educate patients, families, and therapists on how to request or obtain an interpreter if needed. In our study, $99.7 \%$ of therapists reported that an interpreter was present during the session when needed, but future work should be done to determine whether language is a barrier to efficacious telerehabilitation.

Therapists also reported minimal technology barriers and a high percentage of clinical care quality and caregiver/parent involvement during telerehabilitation treatment sessions. Therapists reported telerehabilitation care to be equal or better than in-person clinical care at $82.5 \%$. In addition, $92.5 \%$ of therapists stated that the caregiver/parent was actively involved and participating during the telerehabilitation session. These results highlight the role that telerehabilitation may play in providing therapists more opportunities to understand the patient's home environment and transfer skills from the therapy gym to everyday context. It also may provide ample opportunity for parent/caregiver coaching to increase therapeutic opportunities at home.

\section{LIMITATIONS AND FUTURE DIRECTIONS}

There are several limitations that should be noted for this study. First, the data from this study only included patients who were being seen for developmental occupational and physical therapies at a large, multisite pediatric hospital system in the Midwest, United States in April 2020. This is important to state as it limits generalizability of the results to other disciplines or hospital systems. Second, due to the study design (retrospective, observational), we cannot comment on the effectiveness of therapies provided through telerehabilitation or statistically compare the differences between patients seen via telerehabilitation and in-person clinical care. Future research should focus on understanding the effectiveness of telerehabilitation compared to in-person clinical care and determine which populations would best be served by each model of care.

\section{CONCLUSIONS}

Telerehabilitation will likely not replace traditional in-person clinical care for all patient populations but should be considered as an important adjunct or additional model of care post-pandemic (American Physical Therapy Association, 2019). The results from this study suggest that implementation of telerehabilitation is feasible, can be offered to a wide diversity of patients and families, and is generally accepted by occupational and physical therapists as an effective model of care for pediatric patients.

\section{REFERENCES}

American Occupational Therapy Association. (2018). Telehealth in occupational therapy. American Journal of Occupational Therapy, 72(Supplement_2), 7212410059. https://doi.org/10.5014/ajot.2018.72S219

American Physical Therapy Association. (2019). Position on telehealth. American Physical Therapy Association. https://www.apta.org/aptaand-you/leadership-and-governance/policies/telehealth

Badawy, S. M., \& Radovic, A. (2020). Digital approaches to remote pediatric health care delivery during the COVID-19 Pandemic: Existing evidence and a call for further research. JMIR Pediatrics and Parenting, 3(1), e20049. https://doi.org/10.2196/20049

Baldwin, P., King, G., Evans, J., McDougall, S., Tucker, M. A., \& Servais, M. (2013). Solution-focused coaching in pediatric rehabilitation: An Integrated model for practice. Physical \& Occupational Therapy in Pediatrics, 33(4), 467-483. 
Ben-Pazi, H., Beni-Adani, L., \& Lamdan, R. (2020). Accelerating telemedicine for cerebral palsy during the COVID-19 Pandemic and beyond. Frontiers in Neurology, 11, 746. https://doi.org/10.3389/fneur.2020.00746

Brophy, P. D. (2017). Overview on the challenges and benefits of using telehealth tools in a pediatric population. Advances in Chronic Kidney Disease, 24(1), 17-21. https://doi.org/10.1053/j.ackd.2016.12.003

Burke, B. L., Hall, R. W., \& Section on telehealth care. (2015). Telemedicine: Pediatric applications. Pediatrics, 136(1), e293-308. https://doi.org/10.1542/peds.2015-1517

Camden, C., Pratte, G., Fallon, F., Couture, M., Berbari, J., \& Tousignant, M. (2020). Diversity of practices in telerehabilitation for children with disabilities and effective intervention characteristics: Results from a systematic review. Disability and Rehabilitation, 42(24), 3424-3436. https://doi.org/10.1080/09638288.2019.1595750

Cramer, S. C., Dodakian, L., Le, V., See, J., Augsburger, R., McKenzie, A., ... National Institutes of Health StrokeNet Telerehab Investigators. (2019). Efficacy of home-based telerehabilitation vs in-clinic therapy for adults after stroke: A Randomized clinical trial. JAMA Neurology. https://doi.org/10.1001/jamaneurol.2019.1604

DD Ingram \& SJ Franco. (n.d.). NCHS urban-rural classification scheme for counties. Vital Health Stat, 2(166).

Dorsey, E. R., \& Topol, E. J. (2016). State of telehealth. The New England Journal of Medicine, 375(2), 154-161. https://doi.org/10.1056/NEJMra1601705

Gloff, N. E., LeNoue, S. R., Novins, D. K., \& Myers, K. (2015). Telemental health for children and adolescents. International Review of Psychiatry, 27(6), 513-524. https://doi.org/10.3109/09540261.2015.1086322

Harris, P. A., Taylor, R., Minor, B. L., Elliott, V., Fernandez, M., O’Neal, L., ...REDCap Consortium. (2019). The REDCap consortium: Building an international community of software platform partners. Journal of Biomedical Informatics, 95, 103208. https://doi.org/10.1016/j.jbi.2019.103208

Harris, P. A., Taylor, R., Thielke, R., Payne, J., Gonzalez, N., \& Conde, J. G. (2009). Research electronic data capture (REDCap)—A metadata-driven methodology and workflow process for providing translational research informatics support. Journal of Biomedical Informatics, 42(2), 377-381. https://doi.org/10.1016/j.jbi.2008.08.010

Lee, A. C., Davenport, T. E., \& Randall, K. (2018). Telehealth physical therapy in musculoskeletal practice. The Journal of Orthopaedic and Sports Physical Therapy, 48(10), 736-739. https://doi.org/10.2519/jospt.2018.0613

Levy, C. E., Silverman, E., Jia, H., Geiss, M., \& Omura, D. (2015). Effects of physical therapy delivery via home video telerehabilitation on functional and health-related quality of life outcomes. Journal of Rehabilitation Research and Development, 52(3), 361-370. https://doi.org/10.1682/JRRD.2014.10.0239

Little, L. M., Pope, E., Wallisch, A., \& Dunn, W. (2018). Occupation-based coaching by means of telehealth for families of young children with Autism Spectrum Disorder. American Journal of Occupational Therapy, 72(2), 7202205020. https://doi.org/10.5014/ajot.2018.024786

Nelson, E.-L., \& Sharp, S. (2016). A review of pediatric telemental health. Pediatric Clinics of North America, 63(5), $913-931$. https://doi.org/10.1016/j.pcl.2016.06.011

Novak, I., Morgan, C., Fahey, M., Finch-Edmondson, M., Galea, C., Hines, A., ...Badawi, N. (2020). State of the evidence traffic lights 2019: Systematic review of interventions for preventing and treating children with cerebral palsy. Current Neurology and Neuroscience Reports, 20(2). https://doi.org/10.1007/s11910-020-1022-z

Olson, C. A., McSwain, S. D., Curfman, A. L., \& Chuo, J. (2018). The current pediatric telehealth landscape. Pediatrics, 141(3). https://doi.org/10.1542/peds.2017-2334

Reifenberg, G., Gabrosek, G., Tanner, K., Harpster, K., Proffitt, R., \& Persch, A. (2017). Feasibility of pediatric game-based neurorehabilitation using telehealth technologies: A case report. The American Journal of Occupational Therapy: Official Publication of the American Occupational Therapy Association, 71(3), 7103190040. https://doi.org/10.5014/ajot.2017.024976

Sauers-Ford, H. S., Hamline, M. Y., Gosdin, M. M., Kair, L. R., Weinberg, G. M., Marcin, J. P., \& Rosenthal, J. L. (2019). Acceptability, usability, and effectiveness: A Qualitative study evaluating a pediatric telemedicine program. Academic Emergency Medicine: Official Journal of the Society for Academic Emergency Medicine, 26(9), 1022-1033. https://doi.org/10.1111/acem.13763

Shigekawa, E., Fix, M., Corbett, G., Roby, D. H., \& Coffman, J. (2018). The current state of telehealth evidence: A rapid review. Health Affairs, 37(12), 1975-1982. https://doi.org/10.1377/hlthaff.2018.05132

Software | Epic. (n.d.). Retrieved September 16, 2020, from https://www.epic.com/software

Tanner, K., Bican, R., Boster, J., Christensen, C., Coffman, C., Fallieras, K., ...Marrie, J. (2020). Feasibility and acceptability of clinical pediatric telerehabilitation services. International Journal of Telerehabilitation, 12(2), 43-52. https://doi.org/10.5195/ijt.2020.6336

Tenforde, A. S., Borgstrom, H., Polich, G., Steere, H., Davis, I. S., Cotton, K., ...Silver, J. K. (2020). Outpatient physical, occupational, and speech therapy synchronous telemedicine: A survey study of patient satisfaction with virtual visits during the COVID-19 pandemic. American Journal of Physical Medicine \& Rehabilitation, 99(11), 977-981. https://doi.org/10.1097/PHM.0000000000001571 
Tenforde, A. S., Hefner, J. E., Kodish-Wachs, J. E., laccarino, M. A., \& Paganoni, S. (2017). Telehealth in physical medicine and rehabilitation: A narrative review. PM \& R: The Journal of Injury, Function, and Rehabilitation, 9(5S), S51-S58. https://doi.org/10.1016/j.pmrj.2017.02.013

Tomines, A. (2019). Pediatric telehealth: Approaches by specialty and implications for general pediatric care. Advances in Pediatrics, 66, 5585. https://doi.org/10.1016/j.yapd.2019.04.005

Wallisch, A., Little, L., Pope, E., \& Dunn, W. (2019). Parent perspectives of an occupational therapy telehealth intervention. International Journal of Telerehabilitation, 11(1), 15-22. https://doi.org/10.5195/ijt.2019.6274

WHO | International Classification of Functioning, Disability and Health (ICF). (n.d.). WHO; World Health Organization. Retrieved September 16, 2020, from http://www.who.int/classifications/icf/en/

Zoom. (2020). Zoom Videocommunications Inc. https://zoom.us/docs/doc/Zoom-Security-White-Paper.pdf 